\title{
Antennas for VHF/UHF personal radio: A theoretical and experimental study of characteristics and performance
}

\section{Andersen, Jørgen Bach; Hansen, Flemming}

\section{Published in:}

I E E E Transactions on Vehicular Technology

Link to article, DOI:

10.1109/T-VT.1977.23707

Publication date:

1977

Document Version

Publisher's PDF, also known as Version of record

Link back to DTU Orbit

Citation (APA):

Andersen, J. B., \& Hansen, F. (1977). Antennas for VHF/UHF personal radio: A theoretical and experimental study of characteristics and performance. I E E E Transactions on Vehicular Technology, 26(4), 349-357. https://doi.org/10.1109/T-VT.1977.23707

\section{General rights}

Copyright and moral rights for the publications made accessible in the public portal are retained by the authors and/or other copyright owners and it is a condition of accessing publications that users recognise and abide by the legal requirements associated with these rights.

- Users may download and print one copy of any publication from the public portal for the purpose of private study or research.

- You may not further distribute the material or use it for any profit-making activity or commercial gain

- You may freely distribute the URL identifying the publication in the public portal 


\title{
Antennas for VHF/UHF Personal Radio: A Theoretical and Experimental Study of Characteristics and Performance
}

\author{
J $\emptyset$ RGEN BACH ANDERSEN, MEMBER, IEEE, AND FLEMMING HANSEN, MEMBER, IEEE
}

\begin{abstract}
A theoretical analysis of the characteristics of small personal radio antennas for the $68-$ to $470-\mathrm{MHz}$ frequency range is given. Representing the human body by a simplified lossy dielectric structure, the influence of the body on the performance of the antenna is investigated in detail, and it is shown how antenna impedance, gain, and radiation patterns can be calculated taking the presence of the body into account. For very short antennas the results indicate that radiation from the body may dominate over the radiation contributed by the antenna itself, and that the presence of the body can increase the antenna efficiency considerably, indicating that even very short antennas may provide acceptable radiation efficiencies. The results of the theoretical work are supported by measurements on practical antennas. Quarterwave and short antennas of the helical type are compared with respect to efficiency and radiation patterns at 80,160 , and $450 \mathrm{MHz}$, and it is demonstrated how the physical length of the antenna affects the antenna performance. The design of a very short and compact personal radio antenna is described.
\end{abstract}

\section{INTRODUCTION}

A NTENNAS for personal radios are traditionally whip or wire antennas with physical dimensions comparable to a quarter of a wavelength. At low-or medium-band frequencies such antennas tend to be uncomfortably long, and with modern pocket-sized radio equipment it is highly desirable that antenna dimensions can be reduced. Reduction of antenna dimensions is generally assumed to result in lower efficiency, but for a personal radio antenna where the body of the person carrying the radio set becomes a part of the radiating structure this will not always be the case. The human body will of course distort the radiation pattern and absorb some of the signal power but will at the same time increase the radiation resistance, and the result may be a significant increase of antenna efficiency.

Several authors have studied personal radio antennas [1] [3] but most of the work, which has hitherto been published, has been confined to experimental investigations of the performance of practical antennas. This paper will analyze personal radio antennas theoretically and describe results of measurements on experimental and practical antennas.

\section{THE HUMAN BODY AS AN ANTENNA}

\section{A. Influence of the Body Based on Quasistatic Considerations}

At the frequencies of interest the human body is in the socalled resonance region, where the dimensions are of the same

Manuscript received April 25, 1977; revised July 15, 1977.

$\mathrm{J}$. Bach Andersen was with the Electromagnetic Institute, Technical University of Denmark, Lyngby, Denmark. He is now at Aalborg University Center, Institute of Electronics Systems, Box 159, 9100 Aalborg, Denmark.

F. Hansen is with Storno A/S, DK-2300 Copenhagen S, Denmark. order of magnitude as the wavelength in free space or in the body. Nevertheless, it is of interest to consider briefly a quasistatic model where the body is assumed to be small compared to external and internal wavelengths. The various mechanisms can be discussed from such a model, which puts us in a better position to understand the results obtained from the more complicated model treated in Appendix A.

Let us first convince ourselves that a piece of lossy dielectric may act as an efficient radiator. Assume a cylinder of length $L$ and cross section $A$ with a constant distribution of polarization currents $J_{D}=j \omega\left(\epsilon_{r}-1\right) \epsilon_{0} E^{i}$, where $\epsilon_{r}$ is the complex relative permittivity of the dielectric and $E^{i}$ is the internal electric field. The polarization currents will radiate exactly like currents on a metallic wire; however, due to the conductivity (or loss tangent) of the medium we will also have absorption. With these assumptions we readily find the ratio between the radiated power and the absorbed power

$$
\eta^{\prime}=\frac{P_{\mathrm{rad}}}{P_{\mathrm{loss}}}=\frac{k_{0}{ }^{3} \cdot V \cdot \epsilon^{\prime}\left(1+\tan ^{2} \delta\right)}{6 \pi \cdot \tan \delta},
$$

where $\epsilon^{\prime}$ is the real part of $\epsilon_{r}, \tan \delta$ the loss tangent, $k_{0}=$ $2 \pi / \lambda$ the wavenumber in free space and $V=L \cdot A$ the volume. In $(1)$ it is assumed that $\epsilon^{\prime}-1 \approx \epsilon^{\prime}$. The efficiency of the dielectric as a radiator is given by

$$
\eta=\frac{P_{\mathrm{rad}}}{P_{\mathrm{rad}}+P_{\mathrm{loss}}}=\frac{\eta^{\prime}}{\eta^{\prime}+1} .
$$

We note that in this approximation only the volume counts, not the length and area. Considering the worst possible case, $\tan \delta=1$, we find

$$
\eta^{\prime}=\frac{1}{3 \pi} \cdot k_{0}^{3} \cdot V \cdot \epsilon^{\prime} .
$$

Taking as an example a body of $L=1 \mathrm{~m}, A=0.03 \mathrm{~m}^{2}, f=$ $150 \mathrm{MHz}$, and $\epsilon^{\prime}=80$, we find an efficiency $\eta$ of 89 percent corresponding to a loss of only $0.5 \mathrm{~dB}$. Thus we may conclude that the losses in the body are not a fundamental obstacle to an efficient antenna system, but the problem is rather one of exciting the currents in the body properly (see also [4]).

The simplest excitation is a small electric dipole, which we place a distance $d$ from a small dielectric sphere, the simplest model of the body (see Fig. 1). The dipole induces a field in the dielectric assumed to be constant. Effectively we now have two dipoles, the antenna and the dielectric, with the following ratio between the two dipole moments:

$$
p=\frac{J_{D} \cdot V}{J \cdot d s}=-\frac{3 k_{0}^{3} V}{4 \pi} \cdot \frac{\left(\epsilon_{r}-1\right)}{\left(\epsilon_{r}+2\right)} \cdot f\left(k_{0} d\right) \cdot e^{-j k_{0} d}
$$




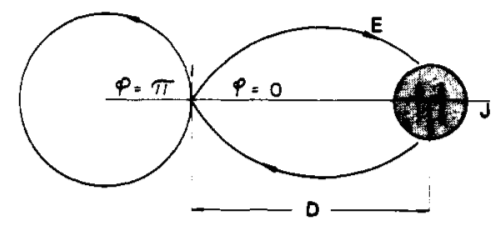

Fig. 1. Electric dipole with dielectric sphere.

where $J d s$ is the dipole moment of the antenna, and

$$
f\left(k_{0} d\right)=\frac{1}{\left(k_{0} d\right)^{3}}+\frac{1}{\left(k_{0} d\right)^{2}}-\frac{1}{k_{0} d} .
$$

Thus we have a two-element array with the following horizontal far field pattern:

$$
F_{h}(\varphi)=1+p \cdot e^{j k_{0} \cdot d \cdot \cos \varphi} .
$$

$\phi=\pi$ is the forward direction assuming that the man has the antenna in front of him, and $\phi=0$ is the backward direction.

In the first rough approximation for $k_{0} d$ small (the antenna close to the body in terms of wavelength) we can neglect the phase factors, and since $\left|\epsilon_{r}\right| \gg 1$ we may also neglect the factor $\left(\epsilon_{r}-1\right) /\left(\epsilon_{r}+2\right)$ leaving us with

$$
F_{h}(\varphi)=1-\frac{3 \cdot V}{4 \pi \cdot d^{3}}<1
$$

Thus for a given current in the dipole the far field is reduced due to the cancelling effect of the polarization currents in the dielectric as sketched in Fig. 1. Note that this effect does not depend on the losses in the dielectric but only on the volume and the distance. In the approximation we are using here the reduction of $F_{h}$ means a reduced radiation resistance, which may have a serious effect on the efficiency if the antenna is small. The impedance relationships will be discussed later.

The first-order effect of the dielectric on the pattern is only on the magnitude, not on the shape. Let us look at secondorder effects where (6) is expanded in terms of $k_{0} d$ and the effect of losses is included. Defining a front-to-back ratio (FTB) as

$$
\mathrm{FTB}=\left|\frac{F(\pi)}{F(0)}\right|^{2},
$$

we arrive at

$$
\mathrm{FTB}=1+\alpha_{\text {loss }}-\alpha_{\text {lens }}
$$

where

$$
\alpha_{\text {loss }}=\frac{27}{4 \cdot \pi^{2}} \cdot \frac{V^{2} k_{0} \tan \delta}{d^{5} \cdot \epsilon^{\prime}\left(1+\tan ^{2} \delta\right)}
$$

and

$$
\alpha_{1 \mathrm{ens}}=\frac{3 k_{0}^{4} \cdot V^{2}}{2 \pi^{2} \cdot d^{2}}
$$

In (9) the following approximations in (6) have been made:

$$
\frac{\epsilon_{r}-1}{\epsilon_{r}+2} \sim 1-\frac{3}{\epsilon_{r}} \sim 1-j \frac{3 \tan \delta}{\epsilon^{\prime}\left(1+\tan ^{2} \delta\right)}
$$

and

$$
e^{-j 2 k_{0} d} \sim 1-j 2 k_{0} d-2\left(k_{0} d\right)^{2} .
$$

It is seen that the losses $(\tan \delta \sim 1)$ produce an FTB larger than one, i.e., we have a shadowing effect from the body due to absorption. The effect is highly distance dependent $\left(d^{-5}\right)$. Equation (9) is of course only valid for $\alpha_{10 s s}$ small, but it has been verified from exact calculations on a lossy sphere that FTB's of more than $15 \mathrm{~dB}$ may result from the close proximity of the antenna to the surface (see below). The other effect due to $\alpha_{\text {lens }}$ reduces the FTB, the body acting as a lens focusing the radiation in the backward direction much the same way as a capacitive director in a Yagi-antenna. When the antenna is close to the body the loss mechanism will dominate over the lens effect. More accurate results for the patterns will be given in the next section.

The fields in the dielectric react back on the antenna and give an impedance change. With the same assumptions as above using the induced EMF method we find (see Appendix A)

$$
\begin{aligned}
\Delta R= & \frac{9}{8 \pi} \cdot R_{0} \cdot k_{0}^{3} \cdot V \\
& \cdot\left(\frac{3 \tan \delta}{\left(k_{0} d\right)^{6} \cdot \epsilon^{\prime} \cdot\left(1+\tan ^{2} \delta\right)}-\frac{4}{3 \cdot\left(k_{0} d\right)^{3}}\right)
\end{aligned}
$$

and

$$
\Delta X=\frac{9}{8 \pi} \cdot \frac{R_{0} \cdot V}{k_{0}^{3} \cdot d^{6}}
$$

where $R_{0}$ is the radiation resistance of the exciting dipole. Only the change in resistance is of interest. It has a positive part and a negative part, with the negative part corresponding to the general reduction of radiation as discussed above. The positive part corresponds to the near field losses in the dielectric and is a serious factor in reducing the gain of the system. Close proximity should be avoided not only for the shadowing effect but also for the low efficiency it creates. The phenomenon is illustrated in Fig. 2, which shows the relative resistance and forward gain as a function of the distance between the dipole and the sphere. The results are exact and based on Mieseries. $\epsilon^{\prime}=80, \sigma=0.5$ correspond to muscle tissue while $\epsilon^{\prime}=8, \sigma=0.05$ correspond to fatty tissue. We may conclude that at this frequency $(f=150 \mathrm{MHz})$ the near-field losses may be neglected when the distance to the surface is greater than $10 \mathrm{~cm}$.

\section{B. Radiation Pattern for Antenna-Man Combination Using Multisphere Model}

At the frequencies of interest the human body is electrically so large that the distribution of polarization currents will not 

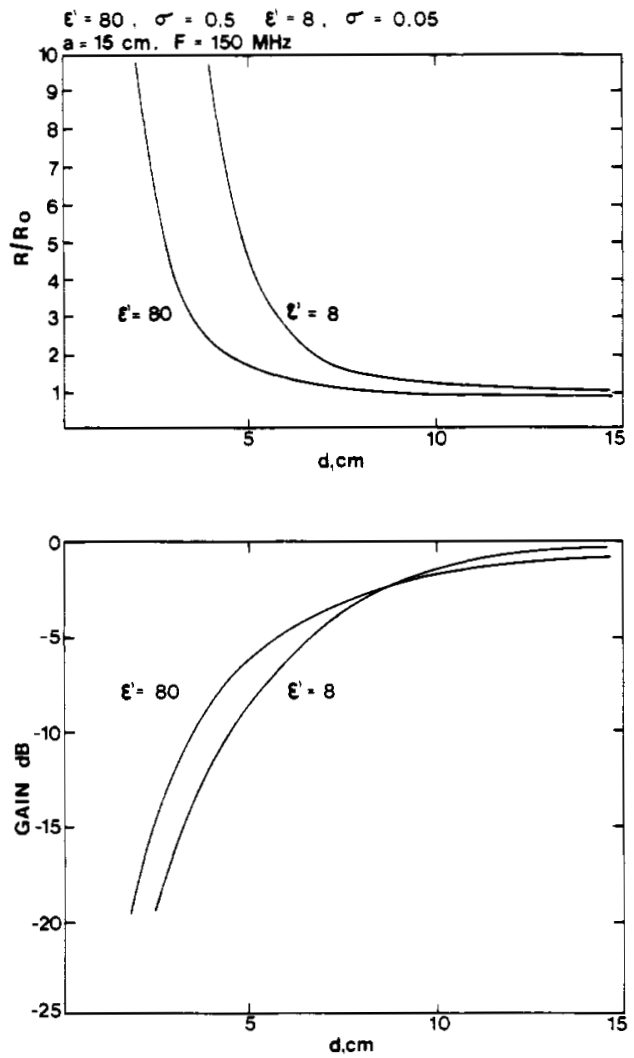

Fig. 2. Dielectric sphere with losses. Exact solution.

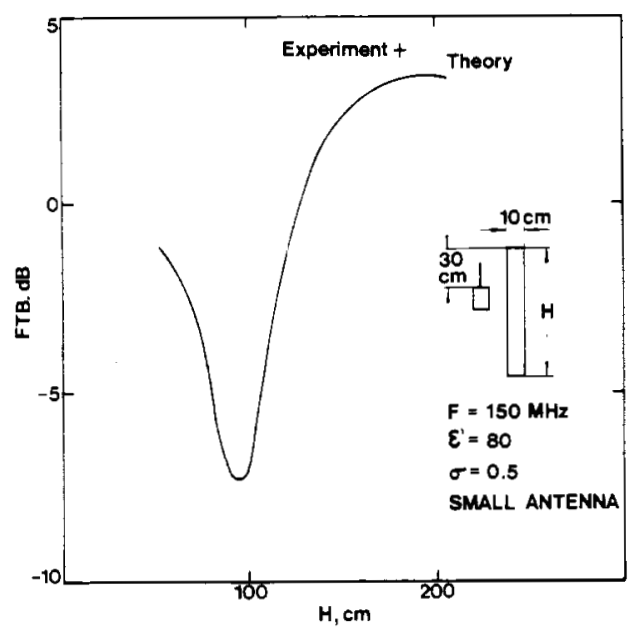

Fig. 3. Front to back ratio as a function of body size.

be uniform. The many-sphere model treated in Appendix A takes this effect into account; instead of one small sphere used in the previous section we now have 17 spheres stacked vertically. Once the current distribution has been found numerically all other parameters such as far field pattern, gain, and impedance are found easily. Fig. 3 gives an example of the use of the multisphere computer program. The figure shows the front-to-back ratio as a function of the height. For small heights we have negative FTB's corresponding to the lens effect mentioned earlier, but for greater heights the situation reverses and for normal body size we have positive FTB's at $150 \mathrm{MHz}$. One experimental result for a small antenna shows

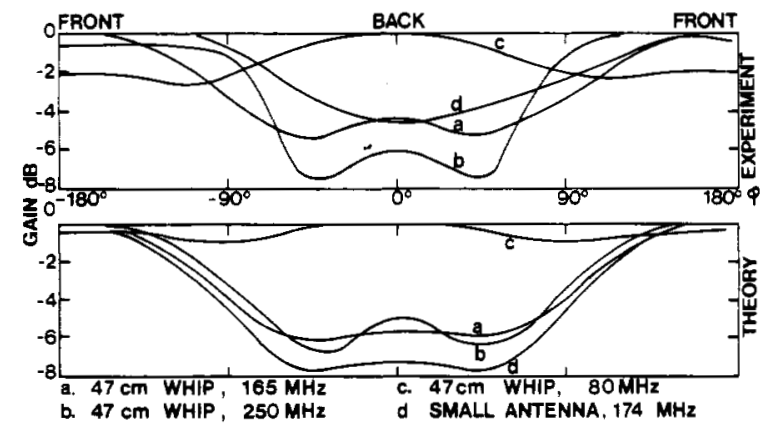

Fig. 4. Experimental and theoretical horizontal patterns. Without hand touching antenna.

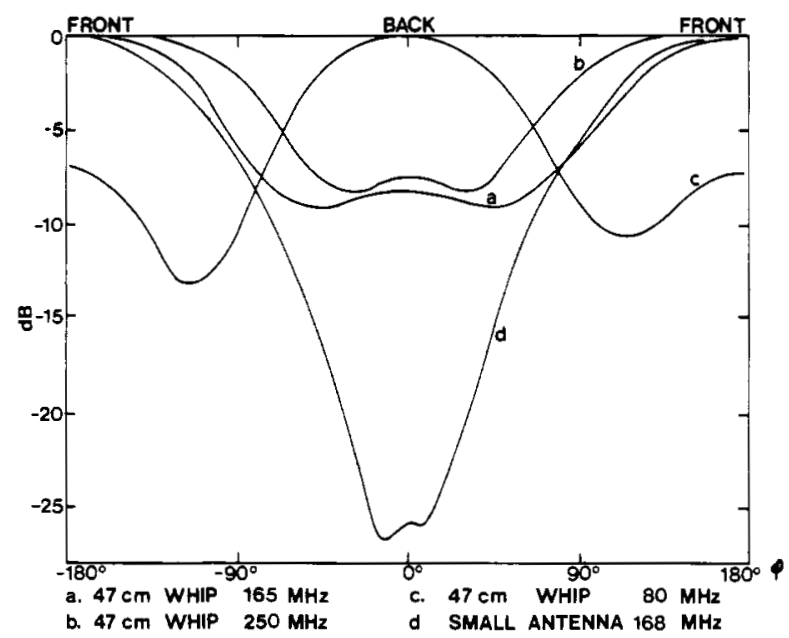

Fig. 5. Measured horizontal patterns with hand touching antenna.

rather good agreement with the theoretical model. A metallic structure would show similar behavior.

Fig. 4 shows both experimental and theoretical horizontal patterns for a whip and a small antenna. The antenna is not touched by the person. No attempt has been made to optimize parameters such that the agreement is improved. At $80 \mathrm{MHz}$ we have the lens effect again, while for the higher frequencies the body acts as a reflector with maximum power in the forward direction. When the hand of the operator is in close contact with the set, the coupling to the body increases as is evident from Fig. 5. All other parameters are the same as in Fig. 4. The variation in the pattern is increased but the general tendencies are the same. No numerical results are available for the handheld case.

Vertical patterns in the forward direction are shown in Figs. 6 and 7. Fig. 6 shows the case where a $50-\mathrm{cm}$ whip is held in front of the body. The measurements are taken in a radioanechoic chamber with the body "floating" in free space. (The theoretical curve is shifted to the left for convenience.) Again we note a difference whether it is with or without hand touching, but in both cases the maximum radiation is slightly upward, about a $20^{\circ}$ elevation angle. An example showing the effect of a lossy ground is shown in Fig. 7 where there is some additional lobing due to the image in the gound; the maximum radiation is now at an angle of $40^{\circ}$ with the horizontal. As we shall see later these idealized patterns may not be very impor. 


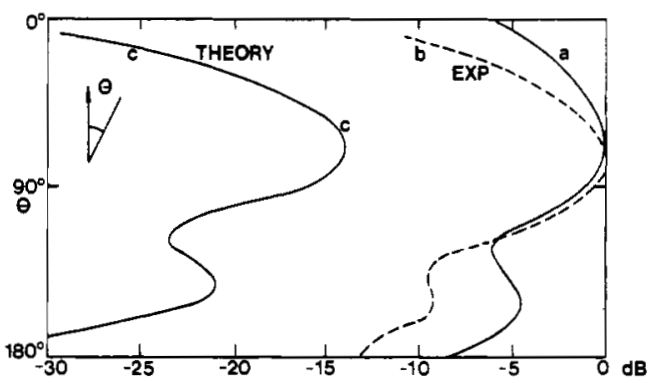

Fig. 6. Vertical patterns in forward direction. $47 \mathrm{~cm}$ ship, $F=165 \mathrm{MHz}$. a) With hand. b) Without hand. c) Theoretical curve (shifted to the left for convenience).
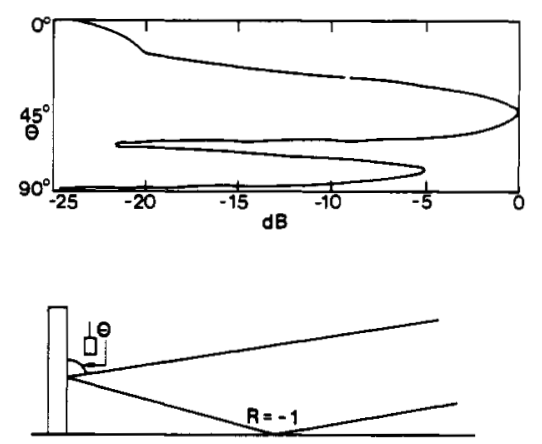

Fig. 7. Vertical pattern in forward direction. Small antenna, $F=165 \mathrm{MHz}$. Reflection coefficient of ground equals -1 .

tant in a realistic situation, but the agreement with the theory indicates the existence and importance of the fields in the body.

\section{Gain and Efficiency of Antenna-Man Combination}

Gain is the product of the directivity and the efficiency (both have been discussed briefly). The theoretical model with the many spheres gives gain directly, relative to a small lossless dipole. Some results are shown in Fig. 8 for a small antenna $15 \mathrm{~cm}$ from the body at a height of $1.5 \mathrm{~m}$. The body has a height of $1.8 \mathrm{~m}$. Forward and backward gain is shown as a function of frequency together with the relative losses in percent. Forward gain is a steadily increasing function of frequency up to about $200 \mathrm{MHz}$ where the low gain at the low frequencies is due partly to a low directivity and partly to high losses. The actual numbers depend on the position of the antenna but may be taken as typical.

This was for the rather academic case of no direct contact with the set. We have seen that the hand affects the patterns, but it may affect the efficiency even more. A rather crude model of the hand would be a cube of side $5 \mathrm{~cm}$ through which the antenna current is assumed to flow. The effect of the hand is then equivalent to an ohmic resistance $R_{\text {hand }}$ in series with the radiation resistance. Assuming a constant current distribution one finds

$$
R_{\mathrm{hand}}=\frac{5 \epsilon^{\prime} \zeta_{0}}{k_{0}|\epsilon-1|^{2}} \Omega
$$

where $\zeta_{0}=120 \pi$ and $k_{0}=2 \pi / \lambda$. For $150 \mathrm{MHz}$ we get $R_{\text {hand }} \sim 8 \Omega$.

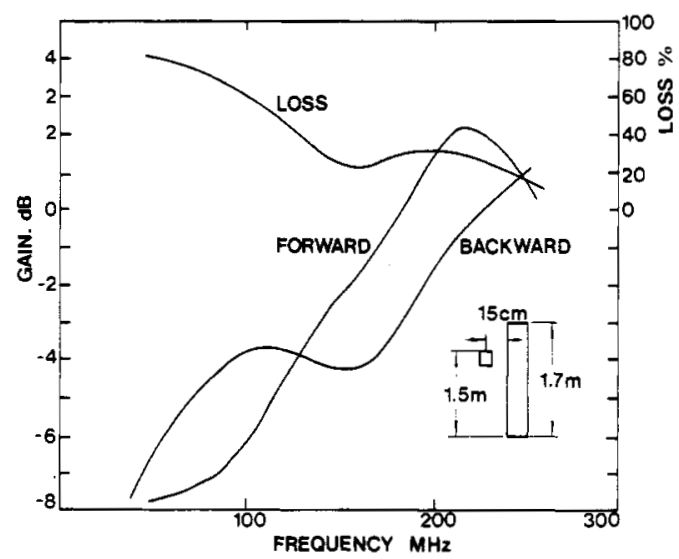

Fig. 8. Forward and backward gain and loss as a function of frequency. Small Antenna.

TABLE I

THEORETICAL AND EXPERIMENTAL GAIN AT $f=165 \mathrm{MHz}$ FOR SMALL ANTENNA AND WHIP

\begin{tabular}{l|l|l|l|l}
\hline & \multicolumn{2}{|l|}{ Smoll Antenna } & \multicolumn{2}{|c}{$\lambda / 4$-wip } \\
\cline { 2 - 5 } & Theory & Exp. & Theory & Exp. \\
\hline Directivity, dB & 2.2 & & 0.9 & \\
Losses in body, dB & 2.2 & & 1.4 & \\
Losses in hand & 3.1 & & 1.1 & \\
Gain, dB & -6.1 & -6 & -1.6 & 0 \\
\hline
\end{tabular}

For a small antenna where $R_{\text {rad }}$ is much smaller than $R_{\text {hand }}$, the hand will act as part of the antenna and couple the energy to the rest of the body. Since the body in itself is reasonably efficient, we can expect a rather efficient antenna system even though the antenna itself may have a low efficiency. This will be the case if

$$
R_{\text {rad }} \ll R_{\text {loss }} \ll R_{\text {hand }}
$$

where $R_{\text {loss }}$ represents the losses in the antenna. Thus we have the rather unusual situation that an inefficient antenna is made efficient by coupling to a lossy structure.

Some typical theoretical and experimental results for $165 \mathrm{MHz}$ are collected in Table I. The small antenna men. tioned is a Hertzian dipole for the numerical results. The experimental small antenna is a $2-\mathrm{cm}$ small fat monopole tuned to resonance by a coil, sitting on top of a $60 \times 60 \times$ $28-\mathrm{mm}$ metal box. It turned out that the gain of the system was close to $-6 \mathrm{~dB}$ independent of the details of the antenna, supporting the argument that the antenna functions more as a coupler than a radiator. Some of the small antennas tested had a gain of -17 to $-18 \mathrm{~dB}$ when isolated from the body but still has $-6 \mathrm{~dB}$ when held in the hand not too close to the body.

\section{DESIGN OF COMPACT ANTENNA}

\section{A. Design Objectives}

The antenna, which will be described, was designed for use on a pocket sized personal radio with dimensions $28 \times 65 \times$ $185 \mathrm{~mm}$. See Fig. 9. This radio set is small enough to be 


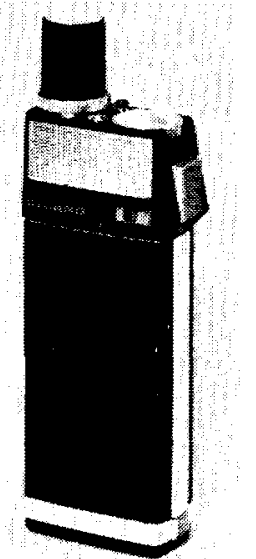

Fig. 9. Personal radio with compact antenna.

TABLE II

TARGET DESIGN SPECIFICATIONS FOR COMPACT ANTENNA

\begin{tabular}{l|c|c|c}
\hline \hline & $M B$ & $\mathrm{HB}$ & UHF \\
\hline Frequency Range, MHz & $68-88$ & $146-174$ & $420-470$ \\
Maximum Duplex Seporation, MHz & 5 & 10 & 10 \\
Minimum Bandwidth (VSWR $<2$ & & & 10 \\
Nominal Impedance, $\Omega$ & 0.5 & 2 & 50 \\
Maximum Input Power, W & 50 & 50 & 5 \\
Minimum Efficiency, relative to \\
quarterwove whip, dB
\end{tabular}

carried conveniently in a shirt pocket, and it was therefore highly desirable that the antenna dimensions could be kept comparably small. The target design specifications for the antenna are summarized in Table II.

\section{B. Antenna Design}

The theoretical analysis indicated that the antenna dimensions may be reduced to a very small fraction of a wavelength before efficiency drops seriously. The limiting parameter will be bandwidth rather than efficiency. This led to the conclusion that a type of radiating element providing inherently large bandwidth and low losses should be chosen and the physical dimensions should be reduced until the minimum acceptable bandwidth was reached. As small bandwidth necessitates tuning it was an additional requirement that it would be possible by simple means to match the antenna to $50 \Omega$ anywhere within the desired frequency range. If transmit/receive switching is needed, electronic control must be possible.

Various types of antenna elements were examined. Fig. 10 shows efficiency versus antenna length at $81 \mathrm{MHz}$ for whip antennas, flexible helically wound antennas, and short thick helical antennas. All measurements were taken with the radio handheld. Similar results were obtained at 150 and $450 \mathrm{MHz}$, and it was concluded that the short fat helical antenna with top load provided the best solution.

The radiating element of the antenna consists of a coil wound on a cylindrical coil former, and the top capacity is provided by a disc with slightly larger diameter than the coil.

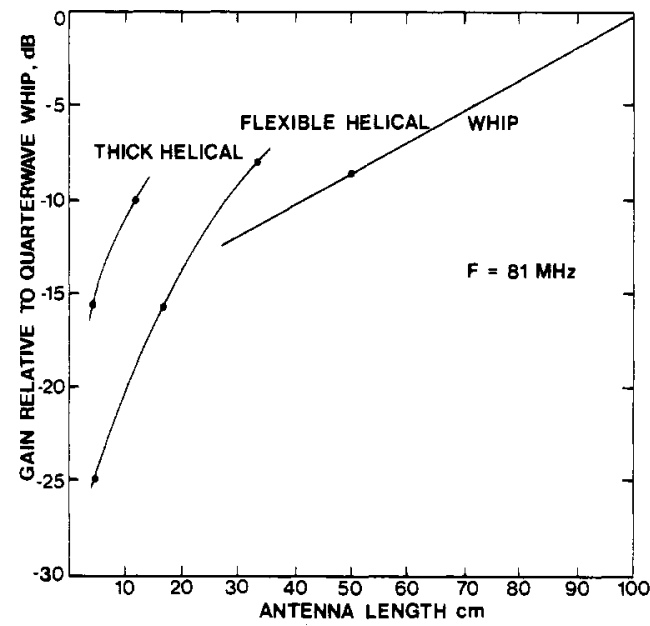

Fig. 10. Efficiency versus antenna length for whip antenna, flexible helical antenna, and thick rigid helical antenna. Handheld.

Tuning elements are placed at the bottom of the antenna and can be accessed through holes in the plastic cap, which is protecting the antenna mechanically.

Transmit/receive switching is accomplished by means of a diode switch, and the antenna can be matched to $50 \Omega$ at two frequencies randomly placed within the band covered. The current controlling the diode switch is supplied via the RF connector. Fig. 9 shows the antenna mounted on the radio.

\section{PERFORMANCE OF COMPACT ANTENNA}

Most of the measurements described in the following were taken with the radio held in the right hand in speaking position $15 \mathrm{~cm}$ from the mouth and with the antenna vertically oriented.

\section{A. Impedance, Bandwidth}

For these measurements a dummy radio was used that could be connected to the impedance measuring equipment via a $50 \Omega$ coaxial cable. Ferrite screening on the outside of the cable was used to suppress RF currents flowing to ground this way.

Figs. 11-13 show typical antenna input impedance versus frequency for MB, HB, and UHF compact antennas. The bandwidths at VSWR $=2$ are summarized in Table III.

\section{B. Radiation Pattern}

Only the horizontal radiation pattern was measured. Measurements took place on an outdoor measuring ground. The antenna was used in the transmit mode, and the radiated signal was measured at a distance of 10 wavelengths while the person carrying the radio was rotating through 360 degrees in steps of 30 degrees. The radio was hand held in normal speaking position. The radiation pattern of a quarter-wave whip was also recorded under the same conditions, and the signal level radiated from this antenna in the forward direction was used as the reference level $(0 \mathrm{~dB})$. Results are depicted in Figs. 1416. The main radiation characteristics are summarized in Table IV. 


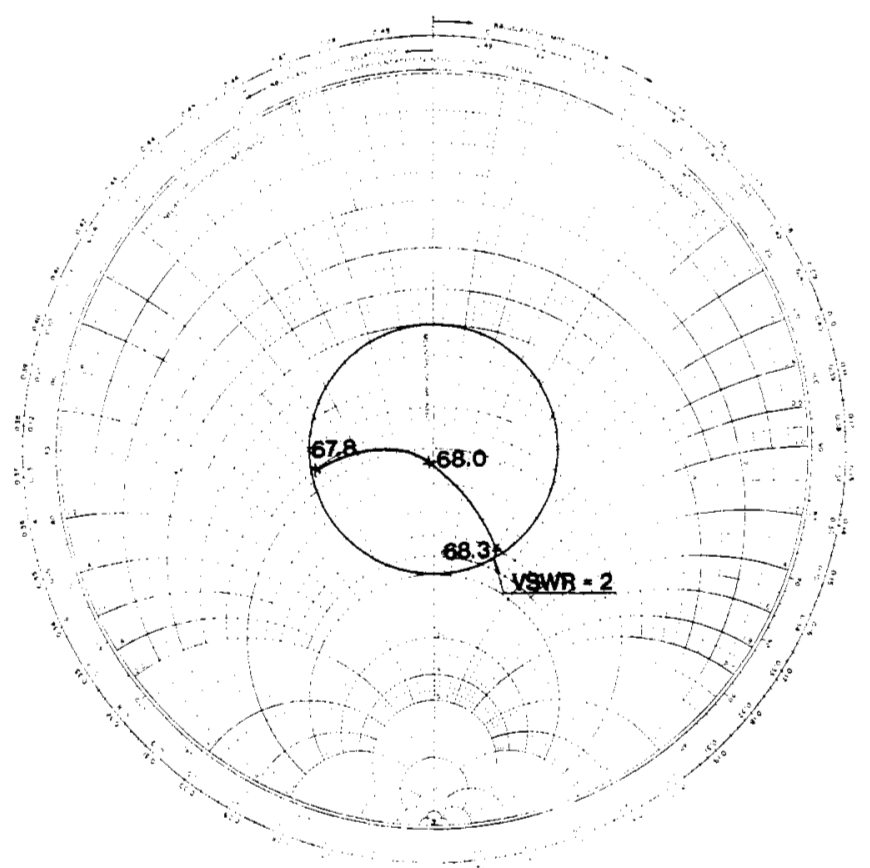

Fig. 11. Input impedance of compact antenna, $80 \mathrm{MHz}$. Handheld.

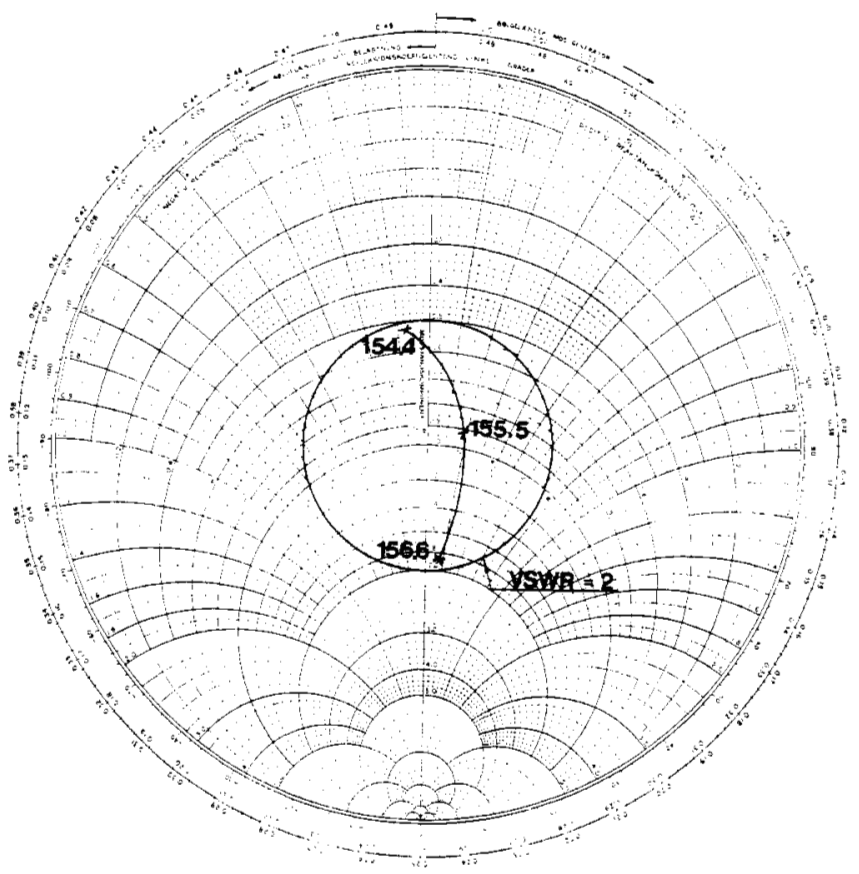

Fig. 12. Input impedance of compact antenna, $150 \mathrm{MHz}$. Handheld.

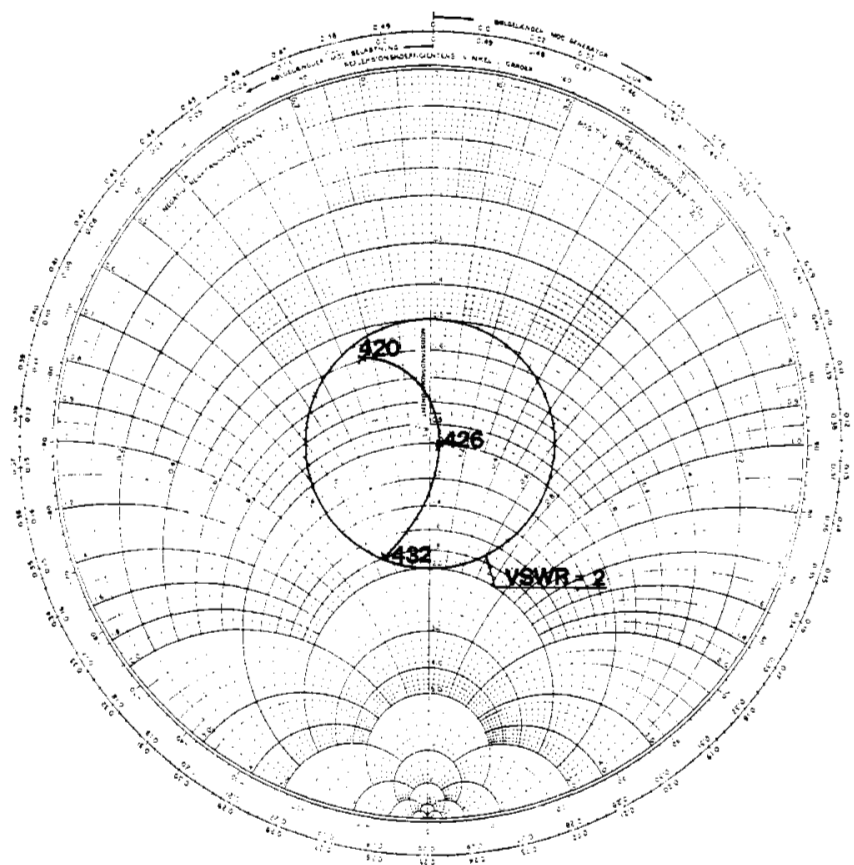

Fig. 13. Input impedance of compact antenna, $450 \mathrm{MHz}$. Handheld.

TABLE III

BANDWIDTH OF COMPACT ANTENNA

\begin{tabular}{|c|c|c|c|}
\hline & $\overline{M B}$ & $\mathrm{HB}$ & UHF \\
\hline Bandwidth (VSWR $<2$ ), & .5 & 2 & 12 \\
\hline
\end{tabular}




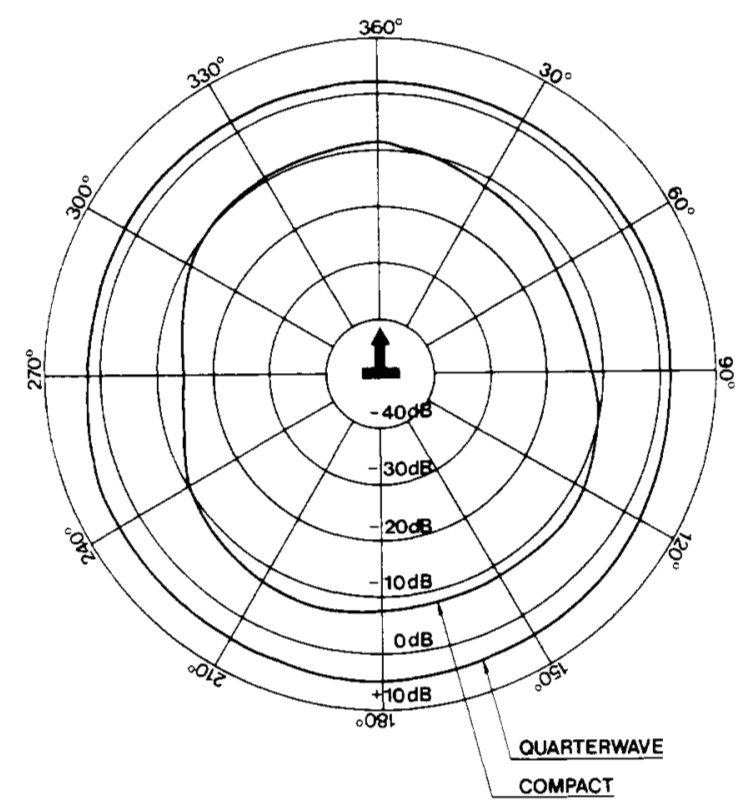

Fig. 14. Radiation pattern of compact antenna, $80 \mathrm{MHz}$. Handheld.

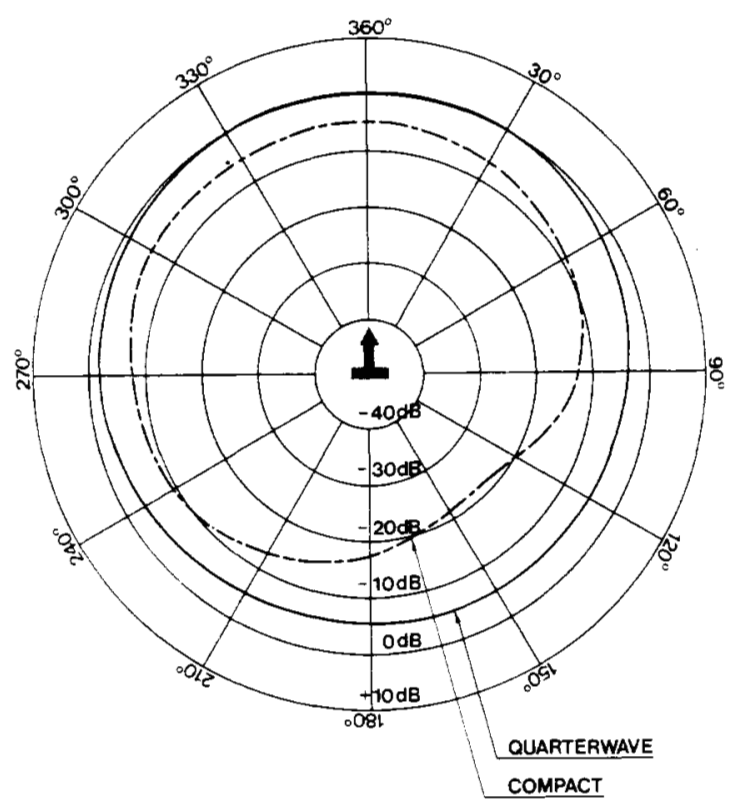

Fig. 15. Radiation pattern of compact antenna, $150 \mathrm{MHz}$. Handheld.

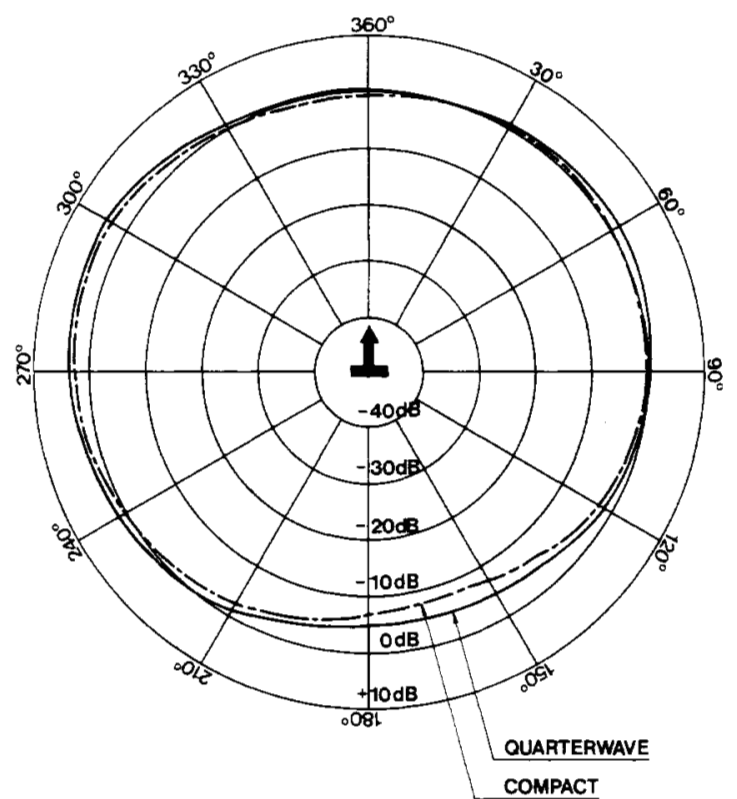

Fig. 16. Radiation pattern of compact antenna, $450 \mathrm{MHz}$. Handheld.

TABLE IV

RADIATION CHARACTERISTICS OF COMPACT ANTENNA

\begin{tabular}{l|c|c}
\hline \hline & $\begin{array}{l}\text { Radiation in max. } \\
\text { direction relative } \\
\text { to } \lambda / 4\end{array}$ & $\begin{array}{c}\text { Max. to min. } \\
\text { ratio }\end{array}$ \\
\hline $80 \mathrm{MHz}$ & -10 & $\mathrm{~dB}$ \\
\hline $150 \mathrm{MHz}$ & -5 & 17 \\
\hline $450 \mathrm{MHz}$ & 0 & 12 \\
\hline
\end{tabular}


TABLE V

EFFICIENCY OF COMPACT ANTENNA FOR DIFFERENT CARRYING MODES

\begin{tabular}{l|c}
\hline & Efficiency relative to $\lambda / 4$ whip \\
\hline Handheld & $-5 \mathrm{~dB}$ \\
\hline Shir: Pocket & $-12 \mathrm{~dB}$ \\
\hline Selt Mount & $-16 \mathrm{~dB}$ \\
\hline
\end{tabular}

Fig. 15 may be compared with Fig. 5 curve $d$ (small antenna, $168 \mathrm{MHz}$ ). The front-to-back ratio is somewhat smaller for the practical antenna, and the whole pattern has been shifted about 45 degrees counterclockwise. Otherwise the agreement is acceptable. These results are in good agreement with the theoretical predictions (see Table I).

With the radio carried in the shirt pocket or clipped onto the belt the efficiencies listed in Table $\mathrm{V}$ were found at $150 \mathrm{MHz}$. Assuming the distance between antenna and body to be 3 to $5 \mathrm{~cm}$ when the radio is worn on the body, according to Fig. 2 the efficiency should drop by 7 to $14 \mathrm{~dB}$. This is in reasonable agreement with the figures in Table $\mathrm{V}$.

\section{Efficiency}

The horizontal radiation pattern gives some information about the antenna, but it does not tell the full story. In many situations the vertical radiation pattern is just as important as the horizontal pattern, and multipath propagation may often cancel the effects of even highly asymmetric radiation patterns.

A more correct measure of the efficiency of a personal radio (or vehicular) antenna can be obtained by averaging the signal levels received while an operator walks along a selected route. When the mean level obtained from a reference antenna on the same route is known, the relative efficiency of the unknown antenna will then be equal to the ratio between the mean levels. Instead of measuring the mean levels directly it is also possible to measure the percentage of time a given signal level has been exceeded and find the relative efficiency as the number of decibels by which the system loss must be increased or reduced in order to obtain the same probability of coverage with the two antennas. A more detailed description of this method is given in Appendix B. Using the latter method, efficiencies relative to a quarter-wave whip are listed in Table VI.

\section{CONCLUSION}

The study of personal antennas described in this paper has shown that significant reduction of the physical size of such antennas is possible before radiation efficiency is seriously affected, and that bandwidth rather than efficiency will be the limiting parameter for the practical design of a very small antenna. The most suitable antenna element for such antennas appears to be a short fat top-loaded helical radiator. Theoretical analysis as well as practical results show that it is possible at $150 \mathrm{MHz}$ with an antenna of this type, only $40 \mathrm{~mm}$ long, to obtain an efficiency better than $-6 \mathrm{~dB}$ relative to a quarter. wave whip while maintaining a bandwidth of $2 \mathrm{MHz}$.
TABLE VI

AVERAGE EFFICIENCY OF COMPACT ANTENNA RELATIVE TO QUARTER-WAVE WHIP. HANDHELD

\begin{tabular}{l|c}
\hline & Efficiency relative to $\lambda / 4$ whip, handheid \\
\hline $80 \mathrm{MHz}$ & $-11.5 \mathrm{~dB}$ \\
\hline $150 \mathrm{MHz}$ & $-5 \mathrm{~dB}$ \\
\hline $450 \mathrm{MHz}$ & $-2 \mathrm{~dB}$ \\
\hline
\end{tabular}

\section{ACKNOWLEDGMENTS}

Jesper Hansen, Technical University of Denmark, Lyngby, is acknowledged for assistance with the experiments. Christian Albertsen made the computer program for the exact calculations for a dipole near a sphere. Leif Mundus-Pedersen, Storno, developed the practical antennas.

\section{APPENDIX A}

\section{NUMERICAL MODEL OF THE HUMAN BODY}

In the computer program the human body is approximated by $M \times N$ closely packed dielectric spheres, $M$ horizontal and $N$ vertical. The diameter of the sphere is $10 \mathrm{~cm}$. The whip is approximated by $L$ current segments exicted by one segment. In each segment of the wire we have a constant unknown current, and in each sphere we have three constant unknown currents (in $x-, y$-, and $z$-direction). The problem is formulated as a matrix problem with all the currents as unknowns, the mutual impedances between the spheres, and the voltage at the excited segment as the source. The fields from the currents in one sphere are assumed to form a dipole field. Once the system of equations is solved we may easily find the system parameters.

The change in impedance $\Delta Z$ equals

$$
\Delta Z=-\frac{\bar{E}^{s} \cdot \bar{h}}{I_{0}}
$$

where $\bar{E}^{s}$ is the scattered field at the feed point from the parasitic currents in the spheres and the other whip elements and $I_{0}$ is the feed current.

$$
P_{\text {in }}=\left(R_{0}+R_{e}(\Delta Z)\right) \cdot\left|I_{0}\right|^{2}
$$

is the input power.

The $\theta$-component of the $E$-field in the far-field is proportional to

$$
\begin{aligned}
e_{\theta}= & \frac{1}{I_{0}} \sum\left(J_{x} \cdot \cos \theta \cdot \cos \varphi+J_{y} \cdot \cos \theta \cdot \sin \varphi-J_{z} \cdot \sin \theta\right) \\
& \cdot \exp \left(j K_{0} \cdot(x \cdot \sin \theta \cdot \cos \varphi+y \cdot \sin \theta \cdot \sin \varphi\right. \\
& +z \cdot \cos \theta))
\end{aligned}
$$

where the summation is over all elements. The gain is proportional to the ratio between the power density in the far-field 


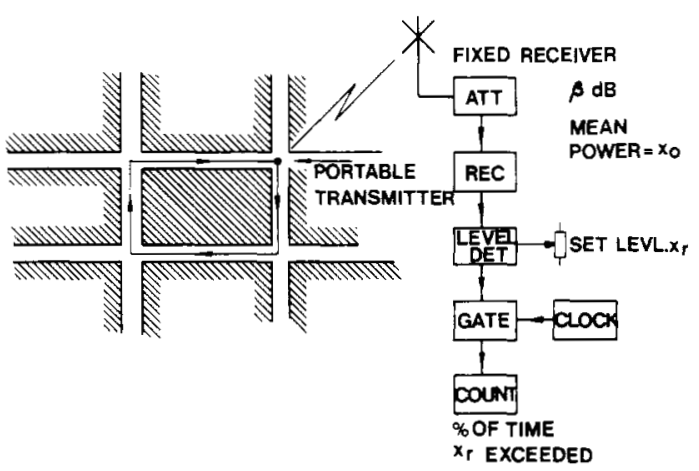

Fig. 17. Measurement of relative antenna efficiency.

and the input power

$$
G=G(\theta, \varphi)=\frac{\left|e_{\theta}\right|^{2} R_{0}}{R_{0}+R_{e}(\Delta Z)} .
$$

Defined in this way $G$ is the gain relative to a vertical Hertzian dipole $\left(\left|e_{\theta}\right|^{2}=1, \Delta Z=0\right)$. The losses in the body $P_{\text {loss }}$ are found by

$$
P_{\text {loss }}=\sigma \int|E|^{2} d V
$$

with efficiency $\eta$ as

$$
\eta=1-\frac{P_{\text {loss }}}{P_{\text {in }}}
$$

and the directivity $D$

$$
D=\frac{G}{\eta}
$$

\section{APPENDIX B}

\section{MEASUREMENT OF RELATIVE ANTENNA EFFICIENCY}

Fig. 17 illustrates the method used. While the pedestrian carrying the portable transmitter walks through the selected route, the percentage of time $P$ during which the signal level received at the base station has exceeded the reference level $X_{r}$ is recorded. The attenuation $\beta$ is so adjusted that a value of $P$ between 0.2 and 0.7 is obtained.

Two sets of measurements are taken, one with the unknown antenna and one with the reference antenna. Assuming Rayleigh fading the probability that $X_{r}$ is exceeded will be

$$
P=P\left(x \geqslant x_{r} \mid x_{0}\right)=e^{-\left(x_{r}\right) /\left(x_{0}\right)}
$$

where $x_{0}$ is the mean power level.
If the values of $P$ found for the unknown and the reference antennas, respectively, are $P_{u}$ and $P_{r}$ we have

$$
P_{u}=e^{-\left(x_{r}\right) /\left(x_{o u}\right)}
$$

and

$$
P_{r}=e^{-\left(x_{r}\right) /\left(x_{o r}\right)} .
$$

From this we find the ratio $a$ between the mean levels

$$
a=\frac{x_{\text {ou }}}{x_{\text {or }}}=\frac{\log P_{r}}{\log P_{u}},
$$

or expressed in $\mathrm{dB}$

$$
\alpha=10 \cdot \log a=10 \cdot \log \frac{\log P_{r}}{\log P_{u}} .
$$

As the same route was used during the two measurements the relative efficiency of the unknown antenna must be

$$
\eta=\eta_{u}-\eta_{r}=\alpha+\left(\beta_{u}-\beta_{r}\right)
$$

or

$$
\eta=10 \cdot \log \frac{\log P_{r}}{\log P_{u}}+\left(\beta_{u}-\beta_{r}\right) .
$$

The use of this method is not restricted to surroundings with pure Rayleigh fading. If propagation conditions are such that the fading will consist of short term Rayleigh fading with lognormal distributed mean level, which is normally the case in urban surroundings, it can be shown [5] that for $x_{R} \ll x_{0}$, $P$ can be approximated by

$$
P=P\left(x \geqslant x_{r} \mid \bar{x}_{0}\right)=e^{-\left(x_{a} / x_{0}\right) e^{\sigma^{2} / M^{2}}}
$$

where $\sigma$ is the standard deviation (bels) of the mean level, and $M=\log e$. Replacing (1) by (8) obviously leads to the same expression (7) for $\eta$.

\section{REFERENCES}

[1] N. H. Shepherd and W. G. Chaney, "Personal radio antennas," IRE Trans. Veh. Commun., vol. VC-10, no. 1, pp. 23-31, Apr. 1961.

[2] Z. Krupka, "The effects of the human body on radiation properties of small-sized communication systems," IEEE Trans. Antennas Propagat., vol. AP-16, no. 2, pp. 154-162, Mar. 1968.

[3] D. K. Belcher, "Human proximity effects on small antennas," Proc. of Carnahan Con. on Crime Countermeasures, pp. 171178, May 1976.

[4] J. Bach Andersen and P. Balling, "Admittance and radiation efficiency of the human body in the resonance region," in Proc. IEEE (Letters), vol. 60, no. 7, pp. 900-901, July 1972.

[5] F. Hansen and F. I. Meno, "Mobile fading-Rayleigh and lognormal superimposed," IEEE Trans. Veh. Technol, vol. VT26 , no. 4, Nov. 1977 


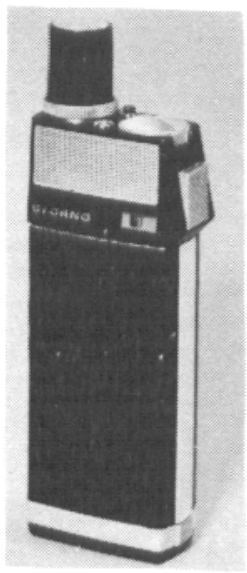

Fig. 9. Personal radio with compact antenna. 